Trauma Surgery \& Acute Care Open

\title{
Preinjury ASA score as an independent predictor of readmission after major traumatic injury
}

\author{
Alexandre Tran, ${ }^{1,2}$ Trinh Mai, ${ }^{3}$ Julie El-Haddad, ${ }^{3}$ Jacinthe Lampron, ${ }^{2}$ Jean-Denis Yelle, ${ }^{2}$ \\ Giuseppe Pagliarello, ${ }^{2}$ Maher Matar ${ }^{2}$
}

\begin{abstract}
- Additional material is published online only. To view please visit the journal online (http://dx.doi.org/10.1136/ tsaco-2017-000128).

'Department of Epidemiology, University of Ottawa, Ottawa, Ontario, Canada 2Division of General Surgery, The Ottawa Hospital, Ottawa, Ontario, Canada

${ }^{3}$ Faculty of Medicine, University of Ottawa, Ottawa, Ontario, Canada
\end{abstract}

Correspondence to Dr Alexandre Tran, Department of Surgery, The Ottawa Hospital, Ottawa, Ontario K1Y 4E9, Canada; aletran@toh.ca

This work was presented at the Trauma Association of Canada Annual Meeting, Halifax, Nova Scotia, Canada, May 2016 and Canadian Surgical Forum, Toronto, on September 2016.

Received 16 August 2017 Revised 23 September 2017 Accepted 8 October 2017

To cite: Tran A, Mai T, El-Haddad J, et al. Trauma Surg Acute Care Open 2017;2:1-4.

\section{ABSTRACT}

Background Patients with trauma have a high predisposition for readmission after discharge. Unplanned solicitation of medical services is a validated quality of care indicator and is associated with considerable economic costs. While the existing literature emphasizes the severity of the injury, there is heterogeneity in defining preinjury health status. We evaluate the validity of the American Society of Anesthesiologists (ASA) Physical Status score as an independent predictor of readmission and compare it to the Charlson Comorbidity Index (CCI).

Methods This is a single center, retrospective cohort study based on adult patients ( $>18$ years of age) with trauma admitted to the Ottawa Hospital from January 1, 2004 to November 1, 2014. A multivariate logistic regression model is used to control for confounding and assess individual predictors. Outcome is readmission to hospital within 30 days, 3 months and 6 months. Results A total of 4732 adult patients were included in this analysis. Readmission rates were $6.5 \%, 9.6 \%$ and $11.8 \%$ for 30 days, 3 months and 6 months, respectively. Higher preinjury ASA scores demonstrated significantly increased risk of readmission across all levels in a dosedependent manner for all time frames. The effect of preinjury ASA scores on readmission is most striking at 30 days, with patients demonstrating a 2.81 (1.88-4.22, $\mathrm{P}<0.0001), 3.59(2.43-5.32, \mathrm{P}<0.0001)$ and 7.52 (4.72-11.99, $P<0.0001)$ fold odds of readmission for ASA class 2, 3 and 4, respectively, as compared with healthy ASA class 1 patients. The ASA scores outperformed the $\mathrm{CCl}$ at 30 days and 3 months.

Conclusions The preinjury ASA score is a strong independent predictor of readmission after traumatic injury. In comparison to the $\mathrm{CCl}$, the preinjury ASA score was a better predictor of readmission at 3 and 6 months after a major traumatic injury.

Level of Evidence Prognostic and Epidemiological Study, Level III.

\section{BACKGROUND}

The patient with trauma presents uniquely complex care needs due to the notable prevalence of mental illness, low socioeconomic status and high rates of recidivism and readmission. ${ }^{12}$ As a result, these patients often face higher rates of major complication as compared with general surgical patients, which in turn leads to considerable economic burden on the healthcare system. ${ }^{3}$ The WHO associates repeated, unplanned solicitation of healthcare services with poor care or lack of coordination of services. ${ }^{4}$ Thus, the ability to identify patients at higher risk for a difficult postdischarge course may allow for more appropriate pre-emptive action. A number of studies have previously evaluated predictors of hospital readmission or re-presentations to the emergency department (ED) after a traumatic injury. ${ }^{5-9}$ These factors include age, race, socioeconomic status, insurance status, existing medical comorbidity, hospital length of stay and discharge disposition.

Much of the existing prediction modeling work in trauma focuses on the injury characteristics and much less so on the patient's baseline health. The need to characterize the patient's premorbid status has been stressed by various authors in the field. ${ }^{10} 11$ However, the methodology for defining comorbidity continues to be highly variable. Several approaches have been described: the presence of major comorbidities (chronic obstructive pulmonary disease or diabetes mellitus), ${ }^{7}$ number of comorbidities ${ }^{6}$ or a global score such as the Charlson Comorbidity Index $(\mathrm{CCI})^{8}$ or the American Society of Anesthesiologists (ASA) Physical Status classification. ${ }^{12}$

While the literature has focused on injury factors and early in-hospital outcomes, there remains a need for early identification of patients at risk for adverse outcomes postdischarge. Therefore, we aim to evaluate the preinjury ASA score as an independent predictor of readmission after a traumatic injury and compare it to the CCI. To our knowledge, there are no studies that have addressed this relationship in the general trauma population.

\section{PATIENTS AND METHODS Population}

This is a single center, retrospective cohort study based on the Ottawa Hospital Trauma Registry database. The Ottawa Hospital (TOH) is a Level 1 Trauma Center and designated as regional adult trauma site for Eastern Ontario in Canada. Registry data are inputted on a daily basis by a dedicated trauma analyst based on a standardized coding protocol implemented province wide as dictated by the Ontario Trauma Advisory Committee. Criteria for inclusion into the database are defined by major traumatic injury requiring trauma code activation or an Injury Severity Score (ISS) greater than or equal to 12. Criteria for local trauma code activation rely on standards dictated by the 2014 Resources for Optimal Care of the Injured Patient. ${ }^{13}$ To limit outcome classification bias for patients presenting back to peripheral hospitals, we used residential postal code classifications to include only patients living within $100 \mathrm{~km}$ of TOH. Given 
our regionalized exclusive trauma system, a relationship exists with surrounding facilities to allow for repatriation of patients requiring readmission back to our hospital, thus improving data capture.

\section{Definition of predictor and outcome variables}

The preinjury ASA score was calculated by the study authors for each patient using a standard protocol to review the clinical documentation available within the medical records at the time of injury. Scoring was based on the ASA classification system presented in the online supplementary figure 1. Any uncertainty regarding scoring was reviewed by a senior author. Previous work by Ringdal et al supports the reliability of the preinjury ASA scale in classifying comorbidity for patients with trauma. ${ }^{10}$ The CCI was captured from the Ottawa Hospital Data Warehouse, a calculation based on a standard 5-year look back of existing medical conditions.

We used an a priori, literature and hypothesis-driven approach to identify potential confounding predictors including age, sex, ISS, mechanism of injury, hospital length of stay, need for operative intervention and disposition on discharge. The outcomes of interest were defined as any readmission within 30 days, 3 months or 6 months to the Ottawa Hospital.

\section{Statistical analysis}

Preliminary analyses were performed using $\mathrm{X}^{2}$ tests for categorical variables and t-tests for continuous variables. The continuous variables did not meet the linearity assumption and as such were recoded to categorical variables based on stratification limits previously illustrated within the literature. We assessed for multicollinearity using variance inflation factors, and variables involved in collinear relationships were excluded based on clinical importance. In accordance with the methodology outlined by the Prognosis Research Strategy guidelines for prediction modeling, we attempted to minimize data-driven bias by avoiding bivariable association testing methods for variable selection. ${ }^{14}$ Instead, a clinical hypothesis-driven approach was used in order for a priori inclusion of all variables in the multivariate logistic regression models for readmission status at 30 days, 3 months or 6 months. We ensured that the recommended sample size threshold of at least 10 events per predictor was met. These considerations minimize the effects of small datasets and results in better model performance at the time of external validation. ${ }^{15}$ The preinjury ASA score was compared with the CCI using receiver operating curve analyses. All analyses were completed using the SAS V.9.4 statistical software.

\section{RESULTS}

We reviewed 5803 patient records during a 10 -year period from January 2004 to November 2014. Following geographical exclusion, we included a total of 4732 adult patients, aged 18 years or older, in our analysis. Readmission rates were $6.5 \%, 9.6 \%$ and $11.8 \%$ for 30 days, 3 months and 6 months, respectively. The median (IQR) time to readmission was 26.2 (8.4-74.0) days. In online supplementary table 1 , we present the characteristics for patients classified as readmission and non-readmission based on preliminary $\mathrm{X}^{2}$ analyses not adjusted for potential confounding. We note a significant relationship for preinjury ASA scores across all levels for prediction of readmission at 30 days, 3 months and 6 months.

In online supplementary table 2 , we present the rates of readmission associated with preinjury ASA levels, noting a trend of increasing risk with higher scores, consistent across all readmission time frames.

In online supplementary table 3, we present the results of our multivariate logistic regression models. We note that higher preinjury ASA scores demonstrated significantly increased risk of readmission across all levels in a dose-dependent manner for all time frames. The effect of preinjury ASA scores on readmission is most striking at 30 days, with patients demonstrating a 2.81fold (1.88-4.22, $\mathrm{P}<0.0001)$, 3.59-fold (2.43-5.32, $\mathrm{P}<0.0001)$ and 7.52-fold (4.72-11.99, $\mathrm{P}<0.0001)$ odds of readmission for ASA class 2, 3 and 4, respectively, as compared with healthy ASA class 1 patients. This effect persists significantly at 3 months and 6 months as well. Discharge to home with supportive care was a significant risk factor whereas discharge to another medical care facility was a significant protective factor for all time frames. Need for multiple operations was a significant predictor at 30 days but not beyond that time frame. Older age was not a significant predictor of readmission at 30 days but was significant at 3 months and 6 months.

In online supplementary table 4 , we present the reasons for readmission for each time frame. Consequences of neurotrauma was the most common reason $(27.0 \%)$ for readmission within 30 days, but this effect appeared to wane over time. In contrast, we note that medical reasons not related to the original traumatic injury accounted for $21.8 \%$ of 30 -day readmissions and increased to $36.0 \%$ of readmissions by 6 months. We note that rates of repeat injury were quite modest accounting for $1.0 \%$, $3.3 \%$ and $5.4 \%$ of readmissions at 30 days, 3 months and 6 months, respectively.

In online supplementary table 5, we present the results of the receiver operating curve comparison between the ASA score and the CCI for prediction of readmission at 30 days, 3 months and 6 months. The prenjury ASA score significantly outperformed the CCI at 30 days and 3 months, though no significant difference was demonstrated at 6 months. We additionally note that at a score cut-off of 2 or higher, the ASA score demonstrated $84.4 \%$ sensitivity and $32.8 \%$ specificity. At a score cut-off of 3 or higher, the ASA score had $63.0 \%$ sensitivity and $56.0 \%$ specificity.

\section{DISCUSSION}

In our single center, retrospective study, we have observed that preinjury ASA score was a significant predictor of readmission after a traumatic injury at 30 days, 3 months and 6 months after discharge. Our multivariate regression analysis demonstrates that patients with higher ASA classification had higher odds of readmission postdischarge with the effect most prominent at 30 days but persisting well into 6 months. In addition, the ASA score outperformed the CCI for prediction of readmission at 30 days and 3 months with no difference noted at 6 months. Our readmission rates of $6.5 \%, 9.6 \%$ and $11.8 \%$ at 30 days, 3 months and 6 months, respectively, are comparable to those presented by Moore et al (5.9\%, 10.9\% and 15.5\%). ${ }^{6}$

To our knowledge, this is the first study to demonstrate the relationship between preinjury ASA scores and the risk for readmission in the general trauma population. This illustrates the importance of capturing and qualifying pre-existing comorbidity in patients with trauma to better predict postdischarge outcomes. The methodology for characterizing baseline health has been highly variable in the literature, and thus the ubiquitous use of a simple, global score would add greatly to the existing prediction modeling work in trauma. 
The CCI is composed of the weighted contributions of various comorbidity categories based on the International Classification of Diseases diagnostic codes available in administrative databases. ${ }^{16}$ It was initially developed to predict 1-year mortality from comorbid illness and is now applied in prognostic models for a variety of illnesses. While the CCI has been studied and validated at an administrative data level as a prognostic indicator for patients with head and neck cancer, ${ }^{17}$ prostate cancer ${ }^{18}$ and lung cancer, ${ }^{19}$ the complexity of its collection and calculation does not allow for bedside application.

Conversely, the ASA classification score has been shown to be a reliable and acceptable predictor of comorbidity and mortality after operation. ${ }^{12}$ In addition, a study comparing the two comorbidity indices for patients with orthopedic compression fractures demonstrated that ASA score outperformed CCI as a predictor for mortality in the surgical population, whereas the CCI outperformed the ASA score in the non-surgical population..$^{20}$ Our findings in this study are similar, noting that the ASA score was a better predictor of readmission at 30 days and 3 months after a major traumatic injury than the CCI. This suggests that the ASA score may be a more appropriately weighted measure of comorbidity when assessing impact on acute illness. Furthermore, the preinjury ASA score was found to be an independent predictor of mortality and the strongest predictor of 30-day readmission for patients with orthopedic trauma. ${ }^{21}$

While we note that the postdischarge outcome literature has traditionally focused on a 30-day window, the recent work by Moore et al has demonstrated that unplanned readmissions persist well past this point. ${ }^{6}$ Therefore, we sought to completely capture the course of recovery of the patient with trauma by studying a more inclusive outpatient period. As we observe a period further away from the time of injury, we expectantly find that predictors related to the patient's injury (ISS, mechanism of injury, need for operation) become less significant and predictors more reflective of a patient's baseline health status (age, preinjury ASA) become more so.

In the early postdischarge period, previous studies suggest that wound complications, non-wound infection, repeat injury, uncontrolled pain are the most common causes of readmission. ${ }^{22}$ However, the proportion of readmissions directly related to the index injury appears to decrease over time. Moore et al demonstrate that only $23.5 \%$ and $30.5 \%$ of readmissions beyond 3 months for patients aged $<65$ and $>65$ years, respectively, were due to complications of the injury. ${ }^{6}$ In our study, we similarly find that medical reasons, unrelated to the index injury, increase over time and account for almost $40 \%$ of readmissions at 6 months.

The implications of these results should be considered as an interesting parallel to the existing work on posthip fracture mortality. It has been demonstrated in multiple studies that despite adjustment for age and comorbid health status, occurrence of a hip fracture leads to independently increased risk of short-term and long-term mortality. ${ }^{23}$ It is hypothesized that the injury either signals or results in a gradual functional decline which 'could result from a lack of mobility and a loss of strength leading to an increase in disability and its associated health consequences'. ${ }^{23}$ Similarly, it is well known that major trauma or critical illness is followed by a hypercatabolic state which leads to profound weight loss, skeletal muscle loss and functional decline. ${ }^{24}$ Therefore, patients with a poor baseline state of health may be more vulnerable to the functional consequences after a traumatic injury and not necessarily a direct complication of the injury itself.

As we approach a time where hospital remuneration depends on outcome measures, the traumatologist must exhibit a high degree of caution when treating elderly, comorbid patients as their baseline health status may perhaps be more influential than the actual injury characteristics in determining unplanned solicitation of healthcare resources. This information can also be used to adjust remuneration, reflecting patients' baseline complexity and need for a higher level of care. Furthermore, in our efforts to ensure the best care for our patients, highrisk individuals should be identified early on in their clinical course and proactive efforts should be undertaken to provide the appropriate in-hospital supports needed to allow for smooth and seamless transitions postdischarge. In our own institution, we intend to incorporate the preinjury ASA score as a simple screening tool when admitting patients with trauma. ${ }^{25}$ Patients that are deemed high risk for readmission would be flagged for early nurse practitioner assessment to analyze appropriateness for multidisciplinary involvement and complex discharge planning. Within our local cohort, a preinjury ASA score cut-off of 3 or higher would have flagged $63.0 \%$ of the 30 -day readmissions. Successful intervention on even a fraction of these patients offers the potential for significant improvement in quality of care and reduction in unplanned resource utilization.

Our findings may be limited by the following considerations. While our logistic regression models were sufficiently powered to assess our primary predictor of interest, we note the limitation of sample size for evaluating other confounding predictors. Variables such as ISS, length of stay and mechanism of injury have been shown to be significant predictors in large cohort studies and have thus been retained in our clinically derived model. In addition, there is certainly the potential for residual confounding bias related to variables we were unable to procure within this study such as need for intensive care unit admission or mechanical ventilation.

Previous works have noted that $34.5 \%$ of readmissions and $25 \%$ of ED re-presentations were to hospitals other than that from their index admission, ${ }^{6}$ which illustrates the challenge of capturing the entirety of postdischarge outcomes in patients with trauma. Therefore, we sought to minimize this potential misclassification bias by restricting our patient population to those living within $100 \mathrm{~km}$ of our trauma center. In addition, our exclusive trauma system for repatriation would allow for more comprehensive capture of patients presenting to surrounding facilities.

\section{CONCLUSION}

The standardized and appropriate classification of baseline comorbidity is important to identify patients most at risk of postdischarge adverse outcomes after a traumatic injury, especially in the long term. In this study, we demonstrate that preinjury ASA score is a strong independent predictor of hospital readmission at 30 days, 3 months and 6 months postdischarge. In comparison to the CCI, the preinjury ASA score was a better predictor of readmission at 30 days and 3 months after a major traumatic injury. In addition, it is a user friendly, easily applicable evaluation that can be performed at the bedside and the existing literature demonstrates strong reliability in evaluating patients with trauma.

Acknowledgements The authors would like to thank Heather Knight for her much appreciated assistance with the Ottawa Hospital Trauma Registry.

Contributors AT and MM conceived the research objective and designed the study protocol. AT, TM, JE-H and MM participated in data extraction. All authors participated in the creation and revision of the manuscript. All authors have read and reviewed the manuscript.

Competing interests None declared. 
Ethics approval Ottawa Health Science Network Research Ethics Board.

Provenance and peer review Not commissioned; externally peer reviewed.

Open Access This is an Open Access article distributed in accordance with the Creative Commons Attribution Non Commercial (CC BY-NC 4.0) license, which permits others to distribute, remix, adapt, build upon this work non-commercially, and license their derivative works on different terms, provided the original work is properly cited and the use is non-commercial. See: http://creativecommons.org/ licenses/by-nc/4.0/

(c) Article author(s) (or their employer(s) unless otherwise stated in the text of the article) 2017. All rights reserved. No commercial use is permitted unless otherwise expressly granted.

\section{REFERENCES}

1 Mullins RJ, Mann NC, Hedges JR, Worrall W, Helfand M, Zechnich AD, Jurkovich GJ. Adequacy of hospital discharge status as a measure of outcome among injured patients. JAMA 1998;279:1727-31.

2 Cameron CM, Purdie DM, Kliewer EV, McClure RJ. Long-term mortality following trauma: 10 year follow-up in a population-based sample of injured adults. J Trauma 2005:59:639-46.

3 Hemmila MR, Jakubus JL, Maggio PM, Wahl WL, Dimick JB, Campbell DA, Taheri PA. Real money: complications and hospital costs in trauma patients. Surgery 2008;144:307-16

4 Cayten CG, Stahl WM, Murphy JG, Agarwarl N, Byrne D, et al. Limitations of the TRISS method. J Trauma 1991;31:471-82.

5 Ladha KS, Young JH, Ng DK, Efron DT, Haider AH. Factors affecting the likelihood of presentation to the emergency department of trauma patients after discharge. Ann Emerg Med 2011;58:431-7.

6 Moore L, Stelfox HT, Turgeon AF, Nathens AB, Le Sage N, Émond M, Bourgeois G, Lapointe J, Gagné M. Rates, patterns, and determinants of unplanned readmission after traumatic injury: a multicenter cohort study. Ann Surg 2014;259:374-80.

7 Morris DS, Rohrbach J, Sundaram LM, Sonnad S, Sarani B, Pascual J, Reilly P, Schwab CW, Sims C. Early hospital readmission in the trauma population: are the risk factors different? Injury 2014;45:56-60.

8 Olufajo OA, Cooper Z, Yorkgitis BK, Najiar PA, Metcalfe D, Havens JM, Askari R, Brat GA, Haider AH, Salim A. The truth about trauma readmissions. Am I Surg 2016:211:649-55.

9 Copertino LM, McCormack JE, Rutigliano DN, Huang EC, Shapiro MJ, Vosswinkel JA, Jawa RS. Early unplanned hospital readmission after acute traumatic injury: the experience at a state-designated level-I trauma center. Am J Surg 2015;209:268-73.
10 Ringdal KG, Skaga NO, Steen PA, Hestnes M, Laake P, Jones JM, Lossius HM. Classification of comorbidity in trauma: the reliability of pre-injury ASA physical status classification. Injury 2013;44:29-35.

11 Skaga NO, Eken T, Søvik S, Jones JM, Steen PA. Pre-injury ASA physical status classification is an independent predictor of mortality after trauma. J Trauma 2007;63:972-8.

12 Wolters U, Wolf T, Stützer H, Schröder T. ASA classification and perioperative variables as predictors of postoperative outcome. Br J Anaesth 1996:77:217-22.

13 Rotondo MF, Cribari C, Smith RS. The American College of Surgeons Committee on Trauma. Resources for optimal care of the injured patient. Chicago: American College of Surgeons, 2014.

14 Steyerberg EW, Moons KG, van der Windt DA, Hayden JA, Perel P, Schroter S, Riley RD, Hemingway H, Altman DG. PROGRESS Group. Prognosis Research Strategy (PROGRESS) 3: prognostic model research. PLoS Med 2013;10:e1001381.

15 Steyerberg EW. Clinical prediction models. A practical approach to development, validation and updating. Berlin, Germany: Springer, 2009.

16 Voskuij T, Hageman M, Ring D. Higher Charlson comorbidity index scores are associated with readmission after orthopaedic surgery. Clin Orthop Relat Res 2014:472:1638-44.

17 Singh B, Bhaya M, Stern J, Roland JT, Zimbler M, Rosenfeld RM, Har-El G, Lucente FE. Validation of the Charlson comorbidity index in patients with head and neck cancer: a multi-institutional study. Laryngoscope 1997;107:1469-75.

18 Fowler JEJ, Terrell FL, Renfroe LD. Co-morbidities and survival of men with localized prostate cancer treated with surgery or radiation therapy. J Urol 1996;156:1714-8.

19 Birim O, Maat AP, Kappetein AP, van Meerbeeck JP, Damhuis RA, Bogers AJ. Validation of the Charlson comorbidity index in patients with operated primary non-small cell lung cancer. Eur J Cardiothorac Surg 2003;23:30-4.

20 Lavelle EA, Cheney R, Lavelle WF. Mortality prediction in a veterbal compression fracture population: the ASA physical status score versus the Charlson Comorbidity Index. Int J Spine Surg 2015;9:63.

21 Sathiyakumar V, Molina CS, Thakore RV, Obremskey WT, Sethi MK. ASA score as a predictor of 30-day perioperative readmission in patients with orthopaedic trauma injuries: an NSQIP analysis. J Orthop Trauma 2015;29:e127-32.

22 Malhotra AK, Martin N, Jacoby M, Tarrant J, Guilford K, Wolfe LG, Aboutanos MB, Duane TM, Ivatury RR. What are we missing: results of a 13-month active follow-up program at a level I trauma center. J Trauma 2009;66:1696-703.

23 Empana JP, Dargent-Molina P, Bréart G. Effect of hip fracture on mortality in elderly women: the EPIDOS prospective study. J Am Geriatr Soc 2004;52:685-90.

24 Service $E$, Anwar F. Nutritional management in critically ill trauma patients is challenging. Trauma 2016;18:231-6.

25. American Society of Anesthesiologists. ASA physical status classification system. 2014 https://www.asahq.org/resources/clinical-information/asa-physical-statusclassification-system. 